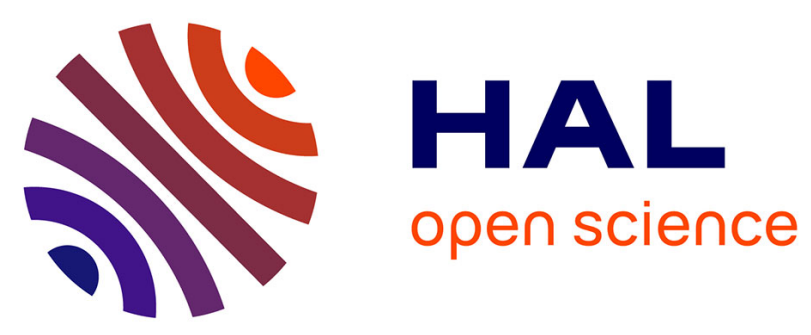

\title{
Experimental characterization of the distribution of resonance widths in chaotic reverberation chambers
}

Elodie Richalot, Ulrich Kuhl, Olivier Legrand, Fabrice Mortessagne, Jean-Baptiste Gros, S Grivet-Talocia

\section{- To cite this version:}

Elodie Richalot, Ulrich Kuhl, Olivier Legrand, Fabrice Mortessagne, Jean-Baptiste Gros, et al.. Experimental characterization of the distribution of resonance widths in chaotic reverberation chambers. 2016 IEEE Metrology for Aerospace (MetroAeroSpace), IEEE, Jun 2016, Florence, Italy. pp.177 181, 10.1109/MetroAeroSpace.2016.7573208 . hal-01397001

\section{HAL Id: hal-01397001 \\ https://hal.science/hal-01397001}

Submitted on 15 Nov 2016

HAL is a multi-disciplinary open access archive for the deposit and dissemination of scientific research documents, whether they are published or not. The documents may come from teaching and research institutions in France or abroad, or from public or private research centers.
L'archive ouverte pluridisciplinaire HAL, est destinée au dépôt et à la diffusion de documents scientifiques de niveau recherche, publiés ou non, émanant des établissements d'enseignement et de recherche français ou étrangers, des laboratoires publics ou privés. 


\title{
Experimental characterization of the distribution of resonance widths in chaotic reverberation chambers
}

\author{
E. Richalot \\ Université Paris-Est, ESYCOM (EA 2552), UPEMLV, ESIEE-Paris, CNAM, 77454 Marne-la-Vallée, France \\ Email: Elodie.richalot@univ-mlv.fr \\ U. Kuhl, O. Legrand, F. Mortessagne \\ Université Nice-Sophia Antipolis, CNRS, Laboratoire de Physique de la Matière Condensée, \\ UMR 7336, Parc Valrose, 06100 Nice, France. \\ J.-B. Gros \\ ISAE, Université de Toulouse, 10 Av. E. Belin BP 54032, 31055 Toulouse, France \\ S. Grivet-Talocia \\ Department of Electronics and Telecommunications, Politecnico di Torino, Torino 10129, Italy
}

\begin{abstract}
Here we investigate how to characterize chaotic reverberation chambers on a spectral basis by experimentally investigating the distribution of resonance widths and comparing it with a theoretical prediction in the weak coupling limit of open chaotic cavities. Those predictions are deduced from the Random Matrix Theory and depend on two parameters, namely the modal overlap and an effective number of open channels. We find a good agreement between those predictions and the distribution of the extracted widths even below the Lowest Useable Frequency in case of sufficiently chaotic reverberation chambers.
\end{abstract}

\section{INTRODUCTION}

Even if normalized criteria have been proposed to characterize the well operation of a reverberation chamber, they are not ideally suited to understand the influence of the physical properties of the cavity on its electromagnetic behavior. In order to master the different influent parameters, it is therefore necessary to characterize the behavior of the cavity in regard to its intrinsic characteristics, in particular in the case of chaotic cavities [1], [2].

The approach we propose is based on a statistical investigation of the resonances of the cavity, as they allow to characterize the behavior of its electromagnetic response. The distribution of the resonance frequencies has been investigated in [3]: we showed the agreement between experimental and theoretical distributions of the nearest-neighbor resonant frequency spacings and how this correlates with the respect of the standard criteria in the case of chaotic cavities. A second aspect of the resonance characteristics is examined here, namely the distribution of their frequency widths.

\section{STUdied REVERBERATION CHAMBERS}

\section{A. Chaotic reverberation chambers}

Based on an analogy between reverberation chambers and chaotic cavities, we showed in our previous works [1], [2] that the field uniformity and isotropy within a reverberation chamber can be improved thanks to simple geometrical modifications inspired from chaotic cavities.

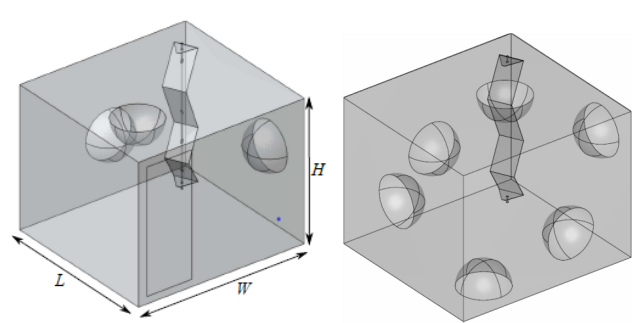

Fig. 1. Commercial reverberation chamber trimmed by (left) three and (right) six metallic hemispheres.

The present paper continues the investigation of the behavior of chaotic reverberation chambers by taking advantage of the theoretical studies on chaotic cavities.

\section{B. Modification of a conventional reverberation chamber}

The commercial reverberation chamber in which measurements were performed consists in a cubic metallic cavity of dimensions $W=2.95 \mathrm{~m}, L=2.75 \mathrm{~m}$ and $H=2.35 \mathrm{~m}$ equipped by a mode-stirrer made of six assembled thin metallic sheets in rotation around a vertical axis and located in a corner (see Fig. 1). The Lowest Useable Frequency (LUF) of this reverberation chamber is given by the manufacturer to be around $300 \mathrm{MHz}$. The corresponding working volume is about $19 \mathrm{~m}^{3}$.

In our previous studies based on simulations and measurements we showed that the field uniformity can be improved in this cavity by adding metallic hemispheres on its metallic walls [1], [2], [4], [5]. The basic idea of this geometrical modification is, in the first instance, to avoid the presence of flat parallel metallic surfaces as they permit regular resonant modes to establish as, for instance, those associated with the multiple reflections between two facing flat parallel surfaces. The presence of defocusing parts on walls also guarantees chaotic motion of the rays in the geometrical limit, therefore making such chambers chaotic [1], [2]. 
Metallic hemispheres of radius $0.4 \mathrm{~m}$ have been fixed on the reverberation chamber walls; as their flat surfaces are in contact with the cavity walls, the electrical continuity is ensured between the cavity and the hemispheres. For the first measurements, three hemispheres were placed on three adjacent walls. This first configuration is presented in Fig. 1 (left). It corresponds to the configuration which was also already investigated in [4] from the point of view of the statistics of the field. As the surface covered by those hemispheres is limited in regard to the surfaces of the metallic walls, three other hemispheres have been added in the second measurement setup presented in Fig. 1 (right), in order to reduce the amount of flat parallel surfaces.

\section{MeAsurement Results}

The scattering parameters between two antennas have been measured through a vector analyzer, (i) between $390 \mathrm{MHz}$ and $410 \mathrm{MHz}$ for the first configuration with three hemispheres, and (ii) between $220 \mathrm{MHz}$ and $270 \mathrm{MHz}$ for both configurations, i.e. below the announced LUF of the bare unmodified reverberation chamber. In the first case (i), 90 positions of the stirrer spaced by 1 degree were used, while 360 positions of the stirrer with an angular step of 1degree were used in the case (ii).

\section{A. Measurement post-processing}

Two different post-processing methods have been applied to the four measured S-parameters, namely the Vector Fitting Algorithm [6] and the harmonic inversion method [7]. The first method consists of a rational approximation of these parameters in the frequency domain, the poles being common to the four $S$-parameters. The second one is very similar, the major difference lying in its application in the time domain. Both resonance extraction methods rely on a decomposition of the frequency S-parameters as a sum of Lorentzians. The imaginary parts of the poles of the decomposition correspond to the cavity resonant frequencies; the complex poles are given by

$$
p_{n}=f_{n}\left(-\frac{1}{2 Q_{n}}+i\right)=-\frac{\Gamma_{n}}{2}+i f_{n}
$$

with $f_{n}$ the $n^{\text {th }}$ resonant frequency, and $Q_{n}$ and $\Gamma_{n}$ its related quality factor and resonance width. For all $S$-parameters we investigated for the present work, the criteria used to validate the extracted values of the poles were: (i) not to be isolated in the parametric view shown in Fig. 3 as a function of the stirrer angle, (ii) to have a residue larger than the noise-level of the measurement. Then, it appeared that, among the values of widths thus generated, the largest values were generally poorly sampled above $1 \mathrm{MHz}$. Indeed, this is due to the fact that some exceptionally large widths are associated to resonances which are not sensitive to mode stirring for some positions. Their fraction never surpasses $2 \%$ of the sample and should probably become less and less probable for much larger samples. This will be investigated in a forthcoming study where motion of the hemispheres and of the antennas will be performed.
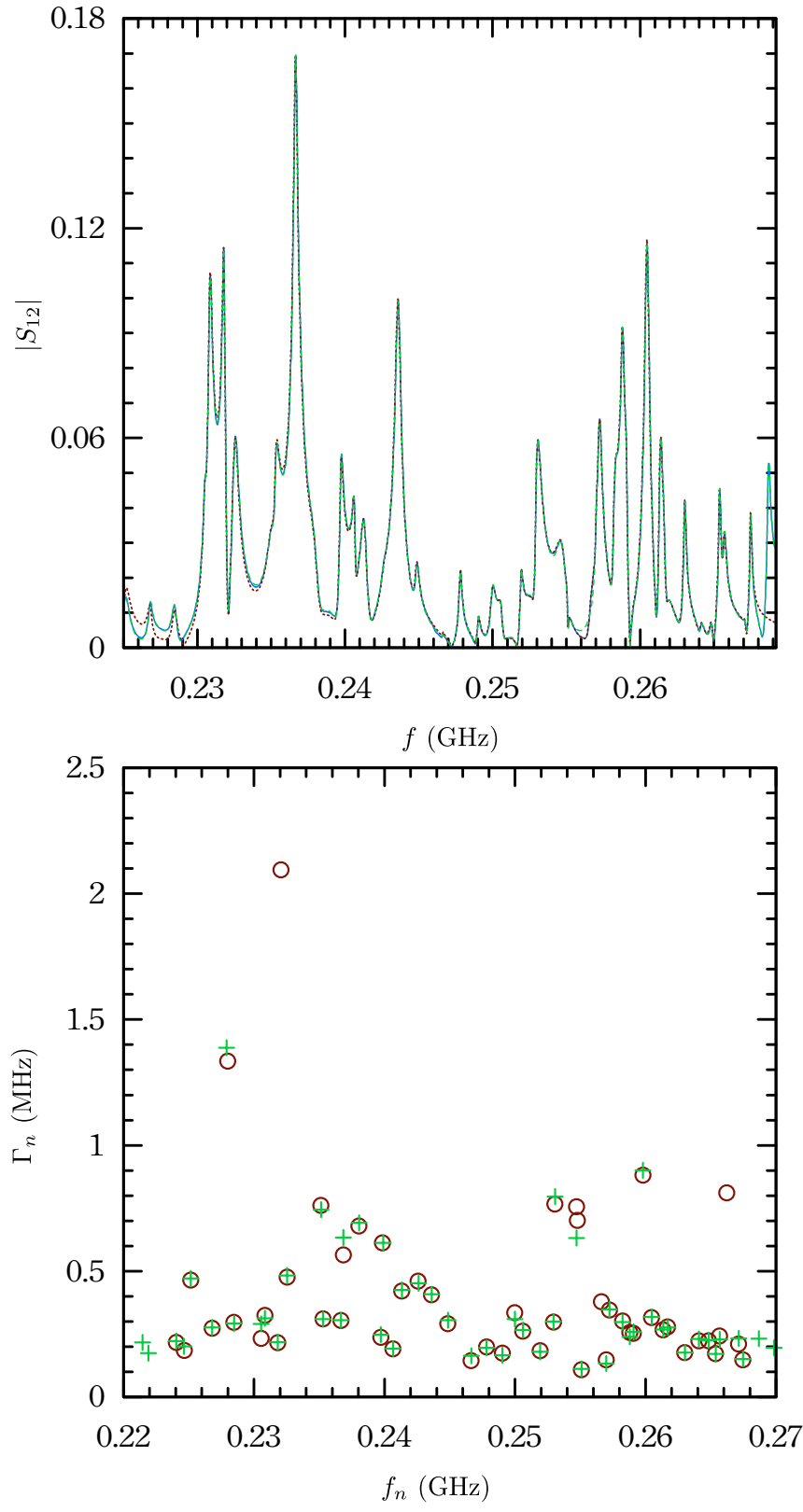

Fig. 2. Top: Transmission coefficient $\left|S_{12}\right|$ (solid line) in the cavity with three hemispheres between 220 and $270 \mathrm{MHz}$ at one stirrer position. Dashed and dotted lines correspond to the reconstruction using the poles obtained by Vector Fitting Algorithm and Harmonic Inversion, respectively. Bottom: Extracted resonance frequencies and widths using both post-processing methods (crosses for Vector Fitting Algorithm and circles for Harmonic Inversion).

In Fig. 2, we present the modulus of transmission amplitude $\left|S_{12}\right|$ (upper part) and the extracted poles obtained using both approaches are shown (lower part) to be equivalent as demonstrated by the reconstruction of $\left|S_{12}\right|$ (dashed and dotted curves).

In the frequency range [220 MHz, $270 \mathrm{MHz}]$, the number of extracted resonance frequencies with their related resonance widths is about 19000 for both cavities when considering the 360 stirrer positions. These numbers are in agreement with the average number of resonances predicted by Weyl's formula 


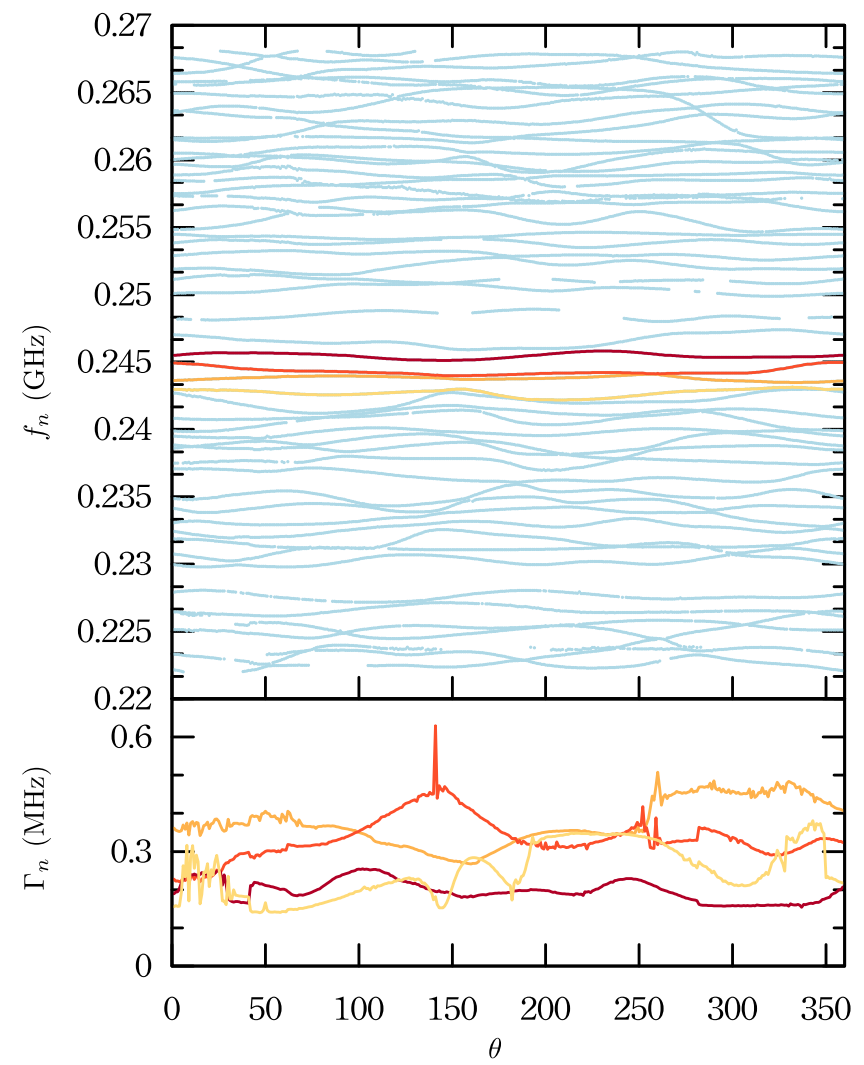

Fig. 3. Top: Variation of the extracted resonance frequencies over the 220$270 \mathrm{MHz}$ frequency band for all the stirrer positions in the cavity with six hemispheres. Bottom: The resonance widths as a function of the stirrer position for 4 resonances. The colored lines refer to the four modes highlighted in the top (using the same color code).

that is in this frequency range equal to 53 (see also Fig. 3).

In the frequency range $[390 \mathrm{MHz}, 410 \mathrm{MHz}]$, around 6300 resonance frequencies and their widths were extracted for the 90 positions of the rotating stirrer. According to Weyl's formula, the average number of modes in this frequency range is of 70 for the cavity with 3 hemispheres.

The distribution of the spacings between adjacent frequencies has been examined in [3] where we showed that the followed law is an indicator of the chaotic behavior of the cavity. The real part of the poles gives the resonance width, which will be the parameter of interest in this paper. The approximate rational expansion also provides information about other parameters characterizing the cavity behavior such as the weight associated to each resonance. Their study could be of great interest but is out the scope of this paper.

Thus, using the above mentioned methods, the resonant frequencies and the related resonance widths have been extracted on the two measured frequency bands for each stirrer position. For both frequency bands and both configurations, all frequency widths have been extracted and their distributions are presented in the following.

\section{B. Distribution of the resonance widths}

It has been demonstrated [5], [8] that for a chaotic system in the weak coupling regime, for weak or moderate modal overlap, the distribution of the resonance widths follows a $\chi^{2}$ law with $M$ degrees of freedom, with $M$ calculated as:

$$
M=2 \frac{\bar{\Gamma}^{2}}{\operatorname{var}\left(\Gamma_{n}\right)}
$$

with $\Gamma_{n}$ the resonance width, the bar denoting the mean and $\operatorname{var}($.$) the variance of the resonance widths. This result relies$ on a random matrix approach where $M$ stands for the number of effective open channels, accounting for all possible loss mechanisms in the cavity. In this approach, the width $\Gamma_{n}$ corresponds to a sum of squares of independent identically distributed Gaussian random variables.

The importance of the system losses is also evaluated through the modal overlap $d$ defined as the ratio of the mean width $\bar{\Gamma}$ over the mean spacing between adjacent resonance frequencies.

It is important to note that the number $M$ of open channels and the modal overlap $d$ are essential parameters for open chaotic systems [5], [8]. Indeed, the coupling strength $\kappa$ of the lossy channels (all assumed to be equivalent) can be expressed in terms of these parameters by:

$$
\kappa=\frac{\pi d}{2 M}
$$

Thus, the weak coupling condition $(\kappa \ll 1)$ may be valid even for a moderate modal overlap if the number of open channels is large enough.

\section{Measurement results below the LUF}

In the frequency range $[220 \mathrm{MHz}, 270 \mathrm{MHz}]$, measurements were performed with cavities loaded by three then six hemispheres.

The complex poles of the four measured $\mathrm{S}$-parameters are determined for each stirrer position. It is to be noticed that the residues associated to the rational functions of the $S$ parameters expansions are also determined, so that the $\mathrm{S}$ parameters expansion can be compared to the measured parameters for verification purpose. As an example, Fig. 2 (top) shows the amplitude of the measured transmission coefficient of the cavity with three hemispheres at the initial position of the mode stirrer, and compares it with the expansions obtained using both post-processing methods. A very good agreement between the three curves is observed.

Fig. 2 (bottom) shows the extracted poles, i.e. eigenfrequencies and widths, for the same configuration. It confirms the agreement between both post-processing methods.

Thus, resonance frequencies and related resonance widths are extracted for each stirrer position. Fig. 3 (top) shows the variation over the stirrer position of all the extracted resonance frequencies in the $[220 \mathrm{MHz}, 270 \mathrm{MHz}]$ in the case of the cavity with six hemispheres, whereas Fig. 3 (bottom) shows the variation of the resonance widths related to the four resonance frequencies between $242 \mathrm{MHz}$ and $247 \mathrm{MHz}$, 


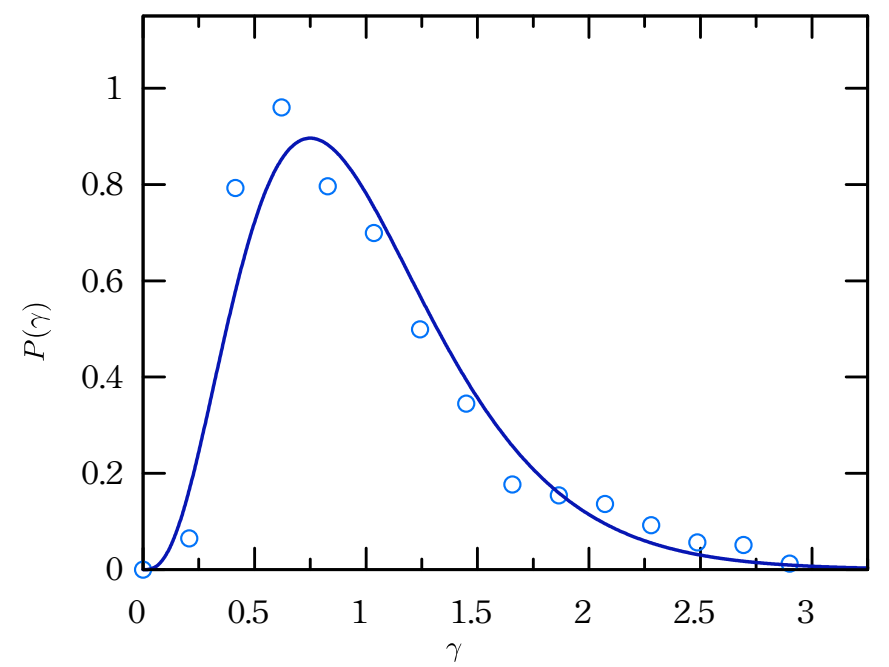

Fig. 4. Probability density function of the normalized resonance widths with three metallic hemispheres in the frequency range $[220 \mathrm{MHz}, 270 \mathrm{MHz}$ (blue circles). Law of Eq. 5 with $M=8$ (blue curve).

which are highlighted by colors. The distribution of all these extracted widths will be examined in the following.

For the three hemispheres case, the mean resonance width is $0.34 \mathrm{MHz}$ and the mean quality factor corresponds to 960 . By excluding $2 \%$ of the widths, corresponding to the largest ones, we obtain a number of degrees of freedom $M=8$ from Eq. 2. With a mean modal overlap $d=0.40$, Eq. 3 leads to the coupling strength $\kappa=0.08$ corresponding to reasonably weak coupling.

Before being compared with the expected analytical law, the resonance widths are normalized according to:

$$
\gamma=\frac{\Gamma}{\bar{\Gamma}}
$$

According to RMT, this normalized resonance width follows the following distribution:

$$
P(x)=\frac{1}{\Gamma(a) b^{a}} x^{a-1} e^{-x / b}
$$

with $a=b^{-1}=M / 2$.

The probability density function of the normalized resonance widths is plotted in Fig. 4 along with the related theoretical law with $M=8$ degrees of freedom. As can be seen, we find an overall agreement but still with non negligible deviations near the maximum.

Therefore, we performed measurements in the same frequency range in the cavity with six hemispheres. The extraction of the resonance properties leads to a mean resonance width of $0.40 \mathrm{MHz}$ and a mean quality factor of 790 . This decrease of the quality factor is due to the increase of the hemisphere surface and the low quality of their metallization.

With a degree of freedom $M=12$, and a mean modal overlap $d=0.44$, one obtains a coupling strength $\kappa=0.06$. The probability density function of the normalized resonance widths is plotted in Fig. 5 along with the related $\chi^{2}$ law. As one can see, the additional three hemispheres made the

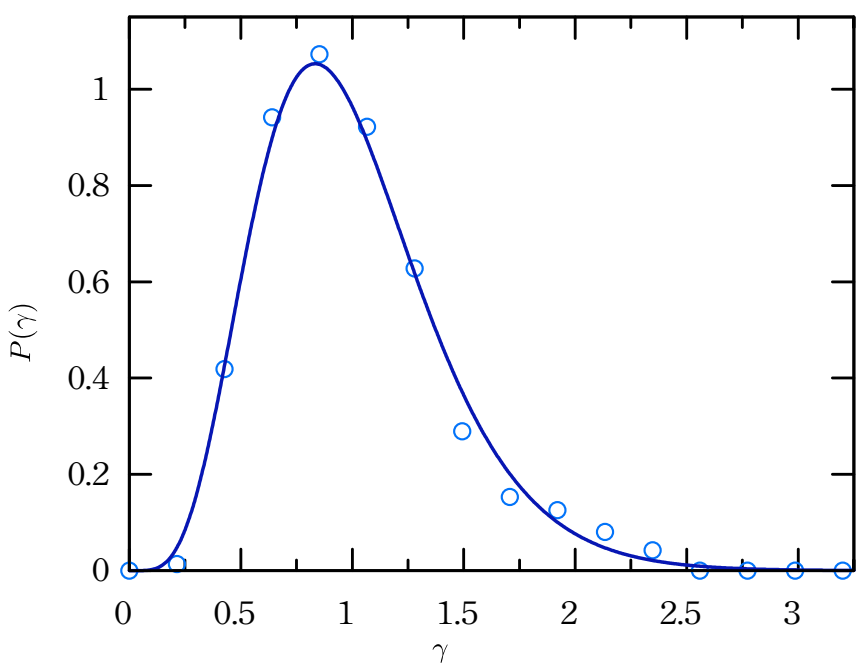

Fig. 5. Probability density function of the normalized resonance widths with six metallic hemispheres in the frequency range [220 MHz, 270 MHz] (blue circles). Law of Eq. 5 with $M=12$ (blue curve).

correspondance between the experiments and the theory much better. This is due to the fact that non-chaotic components are better discarded in the low frequency regime. The better agreement is coherent with our previous studies showing a better chaoticity of the cavity with six hemispheres compared to the one with three hemispheres when considering the spatial field amplitude distribution [9].

\section{Measurement results above the LUF}

In the frequency range [390 MHz, $410 \mathrm{MHz}$, a sample of about 6300 widths was used, yielding a mean width of 0.29 $\mathrm{MHz}$, corresponding to mean quality factor around 1500 .

The probability density function of the normalized resonance widths is plotted in Fig. 6 along with the related $\chi^{2}$ law with $M=20$ degrees of freedom, as deduced from Eq. 2. A good agreement is observed, which indicates that this frequency range corresponds to a weak coupling regime $(\kappa \approx 0.07)$ in spite of a moderate mean modal overlap $d=0.89$ [4]. Here again, our results are coherent with our previous findings. The deviations seen in Fig. 4 have vanished due to the stronger modal overlap.

\section{CHARACTERIZATION OF THE ELECTRIC FIELD DISTRIBUTION IN A CHAOTIC CAVITY}

It has been shown [5] that in a chaotic cavity the normalized amplitude of the field Cartesian components defined in Eq. 6 presents the probability distribution of Eq. 7, with $\rho$ the phase rigidity related to the system losses and defined in Eq. 8.

$$
\begin{gathered}
\mathcal{E}_{a}=\frac{\left|E_{a}\right|}{\sqrt{\left\langle\left|E_{a}\right|^{2}\right\rangle}} \\
P\left(\mathcal{E}_{a}, \rho\right)=\frac{2 \mathcal{E}_{a}}{\sqrt{1-|\rho|^{2}}} \exp \left[-\frac{2 \mathcal{E}_{a}}{\sqrt{1-|\rho|^{2}}}\right] I_{0}\left[\frac{|\rho| \mathcal{E}_{a}^{2}}{1-|\rho|^{2}}\right]
\end{gathered}
$$




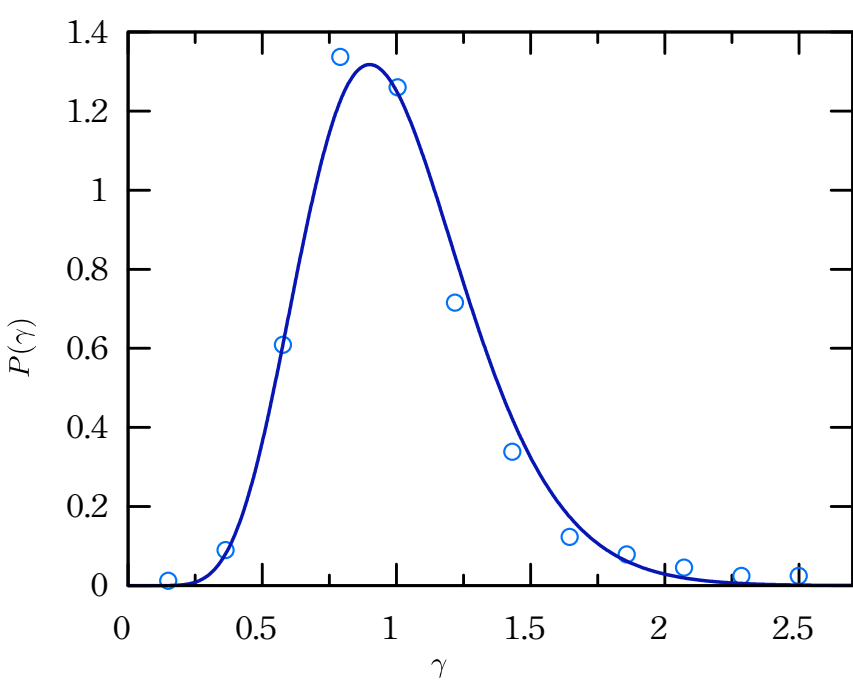

Fig. 6. Probability density function of the normalized resonance widths with three metallic hemispheres in the frequency range $[390 \mathrm{MHz}, 410 \mathrm{MHz}$ ) (blue circles). Law of Eq. 5 with $M=20$ (blue curve).

$$
\rho=\frac{\int_{V} \vec{E} \cdot \vec{E} \mathrm{~d} \vec{r}}{\int_{V}\|\vec{E}\|^{2} \mathrm{~d} \vec{r}}
$$

Once its number of effective open channels $M$ and their coupling strength $\kappa$ are known, the statistical behavior of any chaotic cavity can be simulated through RMT simulations taking the losses into account. In particular, we showed that the distribution of the phase rigidity only depends on the mean modal overlap $d$ as long as this parameter remains weak or moderate [5].

Thus, in the weak coupling and weak modal overlap limit, the distribution of the field Cartesian components of a chaotic cavity is known once its number of effective open channels along with the modal overlap have been determined. As these two parameters can be obtained through measurements at one single antenna location as opposed to classical measurements of field distribution, this approach is of great interest to reduce measurement time and human handling.

\section{CONCLUSION}

We have here pursued the investigation of statistical features of the resonance spectra of chaotic reverberation chambers. Like the distribution of spacings, the distribution of resonance widths can alternatively be used to characterize the degree of chaoticity of the cavity, relying on a model of open chaotic cavities based on the Random Matrix Theory. This model depends on two parameters, namely the modal overlap and an effective number of coupling channels, which account for dissipation in the cavity.The prediction for the distribution of widths corresponds to an analytical law if the coupling strength of the channels is weak.

We have experimentally studied different configurations of chaotic reverberation chambers for different frequency ranges (close to or above the LUF) and by comparing the thus obtained width distributions with the theoretical distribution, we could extract the modal overlap and the number of open channels, which completely characterize the universal statistical behavior of the response of a chaotic reverberation chamber [5]. Results were given here in the case of two different chaotic reverberation chambers and in two frequency bands, one below the expected LUF and the other one above it. This kind of characterization could be used to evaluate the LUF of a chaotic chamber since it allows to avoid a long measurement process that requires field measurements over a stirrer rotation at eight locations of the working volume.

\section{ACKNOWLEDGMENT}

The authors would like to thank the ANR for funding via the project CAOREV.

\section{REFERENCES}

[1] J.-B. Gros, O. Legrand, F. Mortessagne, E. Richalot, and K. Selemani, "Universal behaviour of a wave chaos based electromagnetic reverberation chamber,' Wave Motion, vol. 51, p. 664, 2014.

[2] K. Selemani, J.-B. Gros, E. Richalot, O. Legrand, O. Picon, and O. Mortessagne, "Comparison of reverberation chamber shapes inspired from chaotic cavities," IEEE Trans. on Electromagnetic Compatibility, vol. 57, p. 3, 2014.

[3] E. Richalot, K. Selemani, J. B. Gros, O. Picon, O. Legrand, S. GrivetTalocia, and F. Mortessagne, "Criterion based on resonant frequencies distributions for reverberation chamber characterization," in Electromagnetics in Advanced Applications (ICEAA), 2015 International Conference on, Sept 2015, pp. 1112-1115.

[4] J. B. Gros, U. Kuhl, O. Legrand, F. Mortessagne, and E. Richalot, "Universal intensity statistics in a chaotic reverberation chamber to refine the criterion of statistical field uniformity," in Metrology for Aerospace (MetroAeroSpace), 2015 IEEE, June 2015, pp. 225-229.

[5] J.-B. Gros, U. Kuhl, O. Legrand, and F. Mortessagne, "Lossy chaotic electromagnetic reverberation chambers: Universal statistical behavior of the vectorial field," Physical Review E, vol. 93, 2016.

[6] S. Grivet-Talocia and M. Bandinu, "Improving the convergence of vector fitting for equivalent circuit extraction from noisy frequency responses," IEEE Transactions on Electromagnetic Compatibility, vol. 48, no. 1, pp. 104-120, Feb 2006.

[7] U. Kuhl, R. Höhmann, J. Main, and H.-J. Stöckmann, "Resonance widths in open microwave cavities studied by harmonic inversion," Phyical Review Letters, vol. 100, 2008.

[8] C. Poli, D. V. Savin, O. Legrand, and F. Mortessagne, "Statistics of resonance states in open chaotic systems: A perturbative approach," Physical Review E, vol. 80, 2009.

[9] J. B. Gros, O. Legrand, F. Mortessagne, U. Kuhl, K. Selemani, E. Richalot, and O. Picon, "Field uniformity in chaotic reverberation chambers: Experimental validation," 2015, talk presented at NiceNottingham Days of Complex Media 2015. 